\title{
SATURATION PROPERTIES OF NUCLEAR MATTER AND CORRELATED NUCLEONS
}

\author{
W. H. DICKHOFF \\ Laboratory of Theoretical Physics, University of Gent, \\ Proeftuinstraat 86, B-9000 Gent, Belgium \\ Department of Physics, Washington University, St. Louis, Missouri 63130, USA \\ E-mail:wimd@wuphys.wustl.edu \\ E. P. ROTH \\ Department of Physics, Washington University, St. Louis, Missouri 63130, USA
}

\begin{abstract}
A brief overview is given of the properties of spectral functions in finite nuclei as obtained from $\left(\mathrm{e}, \mathrm{e}^{\prime} \mathrm{p}\right)$ experiments. Based on recent experimental data from this reaction it is argued that the empirical value of the saturation density of nuclear matter is dominated by short-range correlations. This observation and the observed fragmentation and depletion of the single-particle strength in nuclei provide the motivation for attempting a self-consistent description of the nucleon spectral functions with full inclusion of short-range and tensor correlations in nuclear matter. Results for these "second generation" spectral functions will be discussed with emphasis on the consequences for the saturation properties of nuclear matter. Arguments are presented to clarify the obscuring role of pionic long-range correlations in this long-standing problem.
\end{abstract}

\section{Introduction}

Considerable progress has been made in probing the limits of the nuclear mean-field picture in recent years. The primary tool in exhibiting these limits in a quantitative fashion has been provided by the $\left(e, e^{\prime} p\right)$ reaction. 13 The qualitative features of the single-particle (sp) strength distribution can be understood by realizing that a considerable mixing occurs between hole states and two-hole one-particle (2h1p) states. This leads to the observed fragmentation pattern which exhibits a single peak for valence hole states near the Fermi energy, albeit with a reduction of the strength by about $35 \%$. 1 , A broadly fragmented strength distribution is observed for more deeply bound states which is related to the strong coupling to nearby $2 \mathrm{~h} 1 \mathrm{p}$ states. For a complete understanding one requires a global depletion of mean-field orbitals which ranges from $10 \%$ in light nuclei to about $15 \%$ in heavy nuclei and nuclear matter. 4 . This depletion effect must be compensated by the admixture of high-momentum components in the ground state. Such high-momentum nucleons have not yet been unambiguously identified experimentally but they do not appear near the Fermi energy

This information about the spectral strength distripution provides new motivation to consider the "energy" or "Koltun" sum rule.60 In principle, a perfect agreement of the theoretical strength with the experimental one, at all energies and all momenta, must yield a correspondingly good agreement for the energy per particle, provided three-body forces are not too important. The importance of the contribution of high-momentum nucleons, which sofar have not been observed directly, to the energy per particle has been pointed out in Ref. 0 . In addition, we will

manch1: submitted to World Scientific on October 24, 2018 
argue in Sect. 2 that the actual value of the nuclear saturation density is dominated by the effects of short-range (and tensor) correlations (SRC). Recent experimental work supports this claim. Based on these considerations, we propose that a renewed study of the nuclear satuation problem is in order. Special emphasis on SRC must be utilized and we will discard all possible contributions of long-range correlations in nuclear matter. First results of this computationally demanding scheme will be discussed in some detail in Sect. 3. The reasons for discarding the contribution of long-range correlations in nuclear matter are discussed in Sect. 4. Finally, some conclusions are presented in Sect. 5 .

\section{General Considerations concerning Saturation of Nuclear Matter}

Absolute spectroscopic factors associated with quasihole states have been recently obtained for a wide range of nuclei.t. experiments can be directly related to the sp Green's function of the system which is given by

$$
\begin{aligned}
G(\alpha, \beta ; \omega) & =\sum_{m} \frac{\left\langle\Psi_{0}^{A}\left|a_{\alpha}\right| \Psi_{m}^{A+1}\right\rangle\left\langle\Psi_{m}^{A+1}\left|a_{\beta}^{\dagger}\right| \Psi_{0}^{A}\right\rangle}{\omega-\left(E_{m}^{A+1}-E_{0}^{A}\right)+i \eta} \\
& +\sum_{n} \frac{\left\langle\Psi_{0}^{A}\left|a_{\beta}^{\dagger}\right| \Psi_{n}^{A-1}\right\rangle\left\langle\Psi_{n}^{A-1}\left|a_{\alpha}\right| \Psi_{0}^{A}\right\rangle}{\omega-\left(E_{0}^{A}-E_{n}^{A-1}\right)-i \eta} .
\end{aligned}
$$

This Lehmann-representation of the Green's function involves the exact eigenstates and corresponding energies of the $A$ - and $A \pm 1$-particle systems. Both the addition and removal amplitude for a particle from (to) the ground state of the system with $A$ particles must be considered in Eq. (11). Only the removal amplitude has direct relevance for the analysis of the $\left(\mathrm{e}, \mathrm{e}^{\prime} \mathrm{p}\right)$ experiments. The spectroscopic factor for the removal of a particle in the sp orbit $\alpha$, while leaving the remaining nucleus in state $n$, is then given by

$$
z_{\alpha}^{n}=\left|\left\langle\Psi_{n}^{A-1}\left|a_{\alpha}\right| \Psi_{0}^{A}\right\rangle\right|^{2},
$$

which corresponds to the contribution to the numerator of the second sum in Eq. (11) of state $n$ for the case $\beta=\alpha$. Another important quantity is the spectral function associated with sp orbit $\alpha$. The part corresponding to the removal of particles, or hole spectral function, is given by

$$
S_{h}(\alpha, \omega)=\sum_{n}\left|\left\langle\Psi_{n}^{A-1}\left|a_{\alpha}\right| \Psi_{0}^{A}\right\rangle\right|^{2} \delta\left(\omega-\left(E_{0}^{A}-E_{n}^{A-1}\right)\right)
$$

and characterizes the strength distribution of the sp state $\alpha$ as a function of energy in the $A-1$-particle system. From this quantity one can obtain the occupation number

$$
n(\alpha)=\int_{-\infty}^{\epsilon_{F}} d \omega S_{h}(\alpha, \omega)=\left\langle\Psi_{0}^{A}\left|a_{\alpha}^{\dagger} a_{\alpha}\right| \Psi_{0}^{A}\right\rangle .
$$

Experiments on ${ }^{208} \mathrm{~Pb}$ result in a spectroscopic factor of 0.65 for the removal of the last $3 s_{1 / 2}$ proton. 8 Additional information about the occupation number of

manch1: submitted to World Scientific on October 24, 2018 
this orbit can be obtained by analyzing elastic electron scattering cross sections of neighboring nuclei. 0 The occupation number for the $3 s_{1 / 2}$ proton orbit obtained from this analysis is about 0.75 which is 0.1 larger than the quasihole spectroscopic factor. 19 A recent analysis of the $\left(\mathrm{e}, \mathrm{e}^{\prime} \mathrm{p}\right)$ reaction on ${ }^{208} \mathrm{~Pb}$ in a wide range of missing energies and for missing momenta below $270 \mathrm{MeV} / \mathrm{c}$ yields information on the occupation numbers of all the more deeply-bound proton orbitals. The data indicate that all these deeply-bound orbits are depleted by the same amount of about $15 \% .10 .11$ The properties of these occupation numbers in ${ }^{208} \mathrm{~Pb}$ suggest that the main effect of the depletion of the mean-field orbitals is due to SRC. This statement is based on the observation that the effect of the coupling of hole states to low-lying collective excitations only affects occupation numbers of states in the immediate vicinity of the Fermi energy 12 and the knowledge that nuclear matter momentum distributions display such global depletion effects due to shortrange and tensor correlations. 13.14 Indeed, the now observed occupation numbers in ${ }^{208} \mathrm{~Pb} 10.11$ were anticipated some time ago based on this information. 3

Based on these observations, we will now argue that the actual value of the empirical saturation density of nuclear matter is dominated by SRC. Elastic electron scattering from ${ }^{208} \mathrm{~Pb}{ }^{15}$ clearly pinpoints the value of the central charge density in this nucleus. By multiplying this number by $A / Z$ one obtains the relevant central density of heavy nuclei, corresponding to 0.16 nucleons $/ \mathrm{fm}^{3}$ or $k_{F}=1.33 \mathrm{fm}^{-1}$. Since the presence of nucleons at the center of a heavy nucleus is confined to $s$ nucleons, and their depletion is dominated by SRC, one may conclude that the actual value of the empirical saturation density of nuclear matter must also be closely linked to the effects of SRC. While this argument is particularly appropriate for the deeply bound $1 s_{1 / 2}$ and $2 s_{1 / 2}$ protons, it continues to hold for the $3 s_{1 / 2}$ protons which are depleted predominantly by shrt-range effects (up to 15\%) and by at most $10 \%$ due to long-range correlations. 6 . 0

The binding energy of nuclei or nuclear matter usually includes only mean-field contributions to the kinetic energy when the calculations are based on perturbative schemes like the hole-line expansion.16 With the presence of high-momentum components in the ground state it becomes relevant to consider the real kinetic and potential energy of thesystem in terms of the sp strength distributions. This result has the general form 6 .

$$
E_{0}^{A}=\left\langle\Psi_{0}^{A}|\hat{H}| \Psi_{0}^{A}\right\rangle=\frac{1}{2} \sum_{\alpha \beta}\langle\alpha|T| \beta\rangle n_{\alpha \beta}+\frac{1}{2} \sum_{\alpha} \int_{-\infty}^{\epsilon_{F}} d \omega \omega S_{h}(\alpha, \omega)
$$

in the case of only two-body interactions. In this equation $n_{\alpha \beta}$ is the one-body density matrix element which can be directly obtained from the sp propagator. Obvious simplifications occur in this result for the case of nuclear matter due to momentum conservation. A delicate balance exists between the repulsive kinetic-energy term and the attractive contribution of the second term in Eq. (5) which samples the sp strength weighted by the energy $\omega$. When realistic spectral distributions are used to calculate these quantities in finite nuclei unexpected results emerge 5 Such calculations for ${ }^{16} \mathrm{O}$ indicate that the contribution of the quasihole states to Eq. (5), comprises only $37 \%$ of the total energy leaving $63 \%$ for the continuum terms that represent the spectral strength associated with the coupling to low-energy $2 \mathrm{~h} 1 \mathrm{p}$

manch1: submitted to World Scientific on October 24, 2018 
states. The latter contributions exhibit the presence of high-momentum components in the nuclear ground state. Although these high momenta account for only $10 \%$ of the particles in ${ }^{16} \mathrm{O}$, their contribution to the energy is extremely important. These results demonstrate the importance of treating the dressing of nucleons in finite nuclei in determining the binding energy per particle. Similar considerations for nuclear matter have been available for some time as well. 17

It is therefore appropriate to conclude that a careful study of SRC including the full fragmentation of the sp strength is necessary for the calculation of the energy per particle in finite nuclei and nuclear matter. This has the additional advantage that agreement with data from the $\left(\mathrm{e}, \mathrm{e}^{\prime} \mathrm{p}\right)$ reaction aimed at these high-momentum components 18 can be used to gauge the quality of the theoretical description. This argument can be turned inside out by noting that an exact representation of the spectroscopic strength must lead to the correct energy per particle according to Eq. (5) in the case of the dominance of two-body interactions. Clearly this perspective can only become complete upon the successful analysis of high-momentum components in the $\left(\mathrm{e}, \mathrm{e}^{\prime} \mathrm{p}\right)$ reaction.18 An appropriate scheme for nuclear matter that is commensurate with these considerations, is provided by the self-consistent calculation of nucleon spectral functions which include, through the self-energy, the contribution of ladder diagrams involving the propagation of these dressedparticles themselves. Some details of this scheme together with some first results 19 will be discussed in the next section.

\section{Self-consistently Dressed Nucleons in Nuclear Matter}

The equation that represents the calculation of the effective interaction in nuclear matter obtained from the sum of all ladder diagrams while propagating fully dressed particles is given here in the partial wave representation

$$
\begin{aligned}
& \left\langle k\left|\Gamma_{L L^{\prime}}^{J S T}(K, \Omega)\right| k^{\prime}\right\rangle=\left\langle k\left|V_{L L^{\prime}}^{J S T}(K, \Omega)\right| k^{\prime}\right\rangle \\
+ & \sum_{L^{\prime \prime}} \int_{0}^{\infty} d q q^{2}\left\langle k\left|V_{L L^{\prime \prime}}^{J S T}(K, \Omega)\right| q\right\rangle g_{f}^{I I}(q ; K, \Omega)\left\langle q\left|\Gamma_{L L^{\prime \prime}}^{J S T}(K, \Omega)\right| k^{\prime}\right\rangle,
\end{aligned}
$$

where $k, k^{\prime}$, and $q$ denote relative and $K$ the total momentum. Discrete quantum numbers correspond to total spin, $S$, orbital angular momentum, $L, L^{\prime}, L^{\prime \prime}$, and the conserved total angular momentum and isospin, $J$ and $T$, respectively. The energy $\Omega$ and the total momentum $K$ are conserved and act as parameters that characterize the effective two-body interaction in the medium. The critical ingredient in Eq. (6) is the noninteracting propagator $g_{f}^{I I}$ which describes the propagation of the particles in the medium from interaction to interaction. For fully dressed particles this propagator is given by

$$
\begin{aligned}
g_{f}^{I I}\left(k_{1}, k_{2} ; \Omega\right) & =\int_{\epsilon_{F}}^{\infty} d \omega_{1} \int_{\epsilon_{F}}^{\infty} d \omega_{2} \frac{S_{p}\left(k_{1}, \omega_{1}\right) S_{p}\left(k_{2}, \omega_{2}\right)}{\Omega-\omega_{1}-\omega_{2}+i \eta} \\
& -\int_{-\infty}^{\epsilon_{F}} d \omega_{1} \int_{-\infty}^{\epsilon_{F}} d \omega_{2} \frac{S_{h}\left(k_{1}, \omega_{1}\right) S_{h}\left(k_{2}, \omega_{2}\right)}{\Omega-\omega_{1}-\omega_{2}-i \eta},
\end{aligned}
$$

where individual momenta $k_{1}$ and $k_{2}$ have been used instead of total and relative momenta as in Eq. (6). The dressing of the particles is expressed by the use of

manch1: submitted to World Scientific on October 24, 2018 
particle and hole spectral functions, $S_{p}$ and $S_{h}$, respectively. The particle spectral function, $S_{p}$, is defined as a particle addition probability density in a similar way as the hole spectral function in Eq. (3) for removal. These spectral functions take into account that the particles propagate with respect to the correlated ground state incorporating the presence of high-momentum components in the ground state. This treatment therefore includes the correlated version of the Pauli principle leading to substantial modifications with respect to the Pauli principle of the free Fermi gas. This fact suggests that this correlated version may also provide a reasonable description at higher densities. The propagator corresponding to the Pauli principle of the free Fermi gas is obtained from Eq. (7) by replacing the spectral functions by strength distributions characterized by $\delta$-functions as follows

$$
\begin{aligned}
& S_{p}(k, \omega)=\theta\left(k-k_{F}\right) \delta(\omega-\epsilon(k)) \\
& S_{h}(k, \omega)=\theta\left(k_{F}-k\right) \delta(\omega-\epsilon(k)) .
\end{aligned}
$$

This leads to the so-called Galitski-Feynman propagator including hole-hole as well as particle-particle propagation of particles characterized by sp energies $\epsilon(k)$. Discarding the hole-hole propagation then yields the Brueckner ladder diagrams. The effective interaction obtained by solving Eq. (6) using dressed propagators can be used to construct the self-energy of the particle. With this self-energy the Dyson equation can be solved to generate a new incarnation of the dressed propagator. The process can then be continued by constructing anew the dressed but noninteracting two-particle propagator according to Eq. (7). At this stage, one can return to the ladder equation and so on until self-consistency is achieved for the complete Green's function which is then legitimately called a self-consistent one.

While this scheme is easy to present in equations and words, it is quite another matter to implement it. The recent accomplishment of implementing this selfconsistency scheme 19 builds upon earlier approximate implementations. The first nuclear-matter spectral functions were obtained for a semirealistic interaction by employing mean-field propagators in the ladder equation.20,21 Spectral functions for the Reid interaction 22 were obtained by still employing mean-field propagators in the ladder equation but with the introduction of a self-consistent gap in the sp spectrum to take into account the pairing instabilities obtained for a realistic interaction.17.23 The first solution of the effective interaction using dressed propagators was obtained by employing a parametrization of the spectral functions 24,25

The current implementation of the self-consistent scheme for the propagator across the summation of all ladder diagrams includes a parametrization of the imaginary part of the nucleon self-energy. Employing a representation in terms of two gaussians above and two below the Fermi energy, it is possible to accurately represent this self-energy as generated by the contribution of relative $S$-waves (and including the tensor coupling to the ${ }^{3} D_{1}$ channel). 19 Self-consistency at a density corresponding to $k_{F}=1.36 \mathrm{fm}^{-1}$ is achieved in about ten iteration steps, each involving a considerable amount of computational effort.19 A discrete version of this scheme is being implemented successfully by the Gent group.26.27 An important result pertaining to this "second generation" spectral functions is related fo the emergence of a common tail at large negative energy for different momenta.19 Such a common tail was previously obtained at high energy 14 in the particle domain as

manch1: submitted to World Scientific on October 24, 2018 


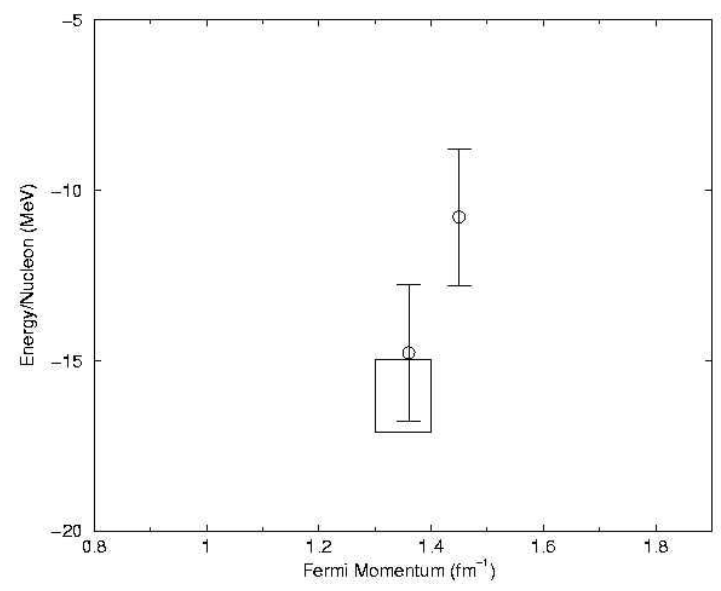

Figure 1. The energy per particle calculated at two different densities from self-consistent spectral functions. The error bars are associated with the lack of self-consistency related to higher order terms in $P$ - and $D$-waves. The saturation density using the Reid potential may possibly be in agreement with the empirical result.

a signature of SRC. This common tail appears to play a significant role in generating some additional binding energy at lower densities compared to conventional Brueckner-type calculations. At present, results for two densities corresponding to $k_{F}=1.36$ and $1.45 \mathrm{fm}^{-1}$ have been obtained. Self-consistency is achieved for the contribution of the ${ }^{1} S_{0}$ and ${ }^{3} S_{1^{-}}{ }^{3} D_{1}$ channels to the self-energy. Higher partial waves are included in the correlated Hartree-Fock contribution. We have obtained additional contributions for $L=2$ and 3 from solutions of the dressed ladder equation after obtaining self-consistency with the dominant $S$ waves. The result for the binding energy have been obtained by averaging the parametrizations of the corresponding self-energies with and without these higher order terms for $L=2$ and 3 partial waves. The difference between these two results then provides us with a conservative estimate of the lack of self-consistency including these terms. This error estimate is included in Fig. 1 for the energy per particle calculated from the energy (Koltun) sum rule in Eq. (5). These results suggest that it is possible to obtain reasonable saturation properties for nuclear matter provided one only includes SRC. The special role of short-range correlations in obtaining this saturating behavior of nuclear matter is illustrated in Fig. 2. In this figure we plot the integrand corresponding to both terms in Eq. (5) as a function of momentum after performing the energy integral over the spectral function for the same densities considered in Fig. 1. It is clear from the figure that at $k_{F}=1.36 \mathrm{fm}^{-1}$ the high-momentum components still provide attractive contributions whereas for $k_{F}=1.45 \mathrm{fm}^{-1}$ a changeover occurs suggesting that at a higher density these high-momentum terms will provide only repulsion. From this analysis it is clear that the expected relevance of SRC in obtaining reasonable saturation properties of nuclear matter is fully confirmed. It remains to relate this observation to the vast body of work on the nuclear-matter saturation problem.

manch1: submitted to World Scientific on October 24, 2018 


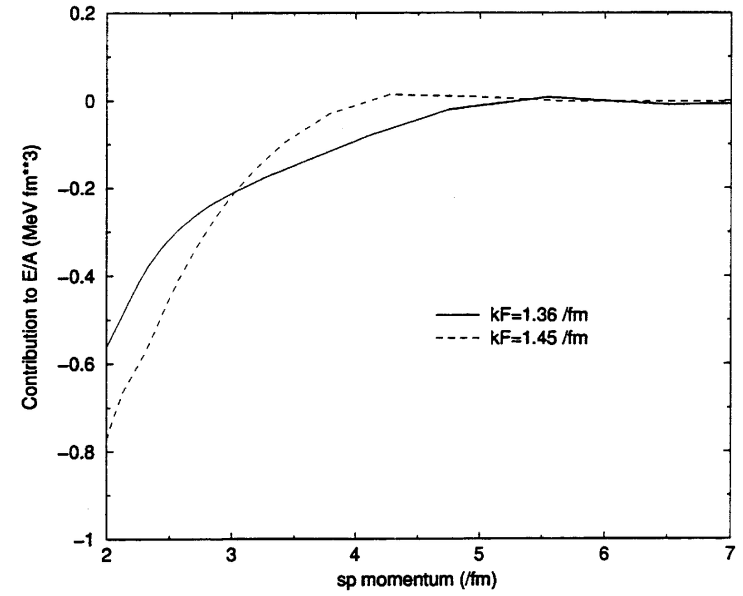

Figure 2. The high-momentum contribution to the energy per particle for $k_{F}=1.36 \mathrm{fm}^{-1}$ (solid) and $1.45 \mathrm{fm}^{-1}$ (dashed). This result illustrates the source of the saturation process when SRC are considered self-consistently.

\section{Discussion of Long-Range Correlations in Nuclear Matter}

We begin this discussion by pointing out the recent success of the Catania group in determining the nuclear saturation curve including three hole-line contributions.16 These calculations demonstrate that a good agreement is obtained at the three hole-line level between calculations that start from different prescriptions for the auxiliary potential. The three hole-line terms obtained in Ref. 16 indicate reasonable convergence properties compared to the two hole-line contribution. One may therefore assume that these results provide an accurate representation of the energy per particle as a function of density for the case of only nonrelativistic nucleons. The obtained saturation density corresponds to $k_{F}=1.565 \mathrm{fm}^{-1}$ with a binding energy of $-16.18 \mathrm{MeV}$. The conclusion appears to be appropriate that additional physics in the form of three-body forces or the inclusion of relativistic effects is necessary to repair this obvious discrepancy with the empirical saturation properties.

Before agreeing with this conclusion it is useful to remember that three hole-line contributions include a third-order ring diagram characteristic of long-range correlations. The agreement of three hole-line calculations with advanced variational calculations 28 further supports the notion that important aspects of long-range correlations are included in both these calculations. This conclusion can also be based on the observation that hypernetted chain calculations effectively include ring-diagram contributions to the energy per particle although averaged over the Fermi sea.29 The effect of these long-range correlations on nuclear saturation properties is not small and can be quantified by quoting explicit results for three- and four-body ring diagrams.30 These results for the Reid potential, including only nucleons, demonstrate that such ring-diagram terms are dominated by attractive

manch1: submitted to World Scientific on October 24, 2018 


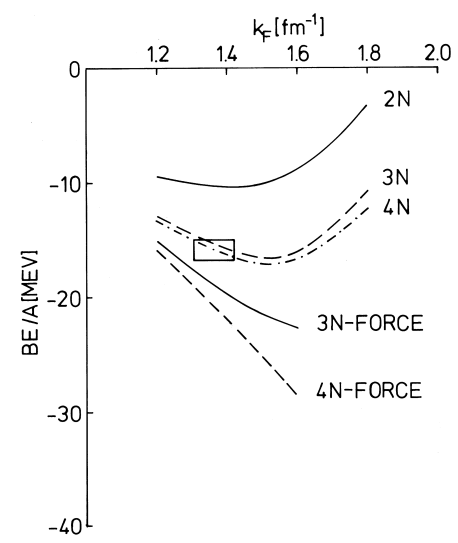

Figure 3. Contribution of three- and four-body ring diagrams involving at least one $\Delta$-isobar configuration, to the energy per particle. These terms can be considered as three- and four-body force contributions when only nucleons are considered. These contributions have been added to the results of Ref. 31 for the Reid interaction.

contributions involving pion quantum numbers propagating around the rings. Furthermore, these contributions increase in importance with increasing density. Including the possibility of the coupling of these pionic excitation modes to $\Delta$-hole states in these ring diagrams leads to an additional large increase in the binding with increasing density 30 This result is illustrated in Fig. 3 where these contributions are added to the hole-line expansion results from Ref. 31. Alternatively, these terms involving $\Delta$-isobars can also be considered as contributions due to three- and four-body forces in the space of only nucleons. The importance of these long-range contributions to the binding energy is related to the possible appearance of pion condensation at higher nuclear density. These long-range pion-exchange dominated contributions to the binding energy appear because of conservation of momentum in nuclear matter. For a given momentum $q$ carried by a pion around a ring diagram, one is able to sample coherently the attractive interaction that exists for values of $q$ above $0.7 \mathrm{fm}^{-1}$. All ring diagrams contribute coherently when the interaction is attractive and one may therefore obtain huge contributions at higher densities which reflect the importance of this collective pion-propagation mode. 32 This is clearly illustrated by the results shown in Fig. 3 .

No such collective pion-degrees of freedom are actually observed in finite nuclei. A substantial part of the explanation of this fact is provided by the observation that in finite nuclei both the attractive and repulsive parts of the pion-exchange interaction are sampled before a build-up of long-range correlations can be achieved. Since these contributions very nearly cancel each other, which is further facilitated by the increased relevance of exchange terms, 33 one does not see any marked effect on pion-like excited states in nuclei associated with long-range pion degrees of freedom even when $\Delta$-hole states are included. 34 It seems therefore reasonable to call into question the relevance of these coherent long-range pion-exchange contributions to the binding energy per particle since their behavior is so markedly different

manch1: submitted to World Scientific on October 24, 2018 
in finite and infinite systems. Clearly, the assertion that long-range pion-exchange contributions to the energy per particle need not be considered in explaining nuclear saturation properties, needs to be further investigated. In practice, this means that one must establish whether pion-exchange in heavy nuclei already mimics the corresponding process in nuclear matter. If this does not turn out to be the case, the arguments for considering the nuclear-matter saturation problem only on the basis of the contribution of SRC will be strenghtened considerably. Furthermore, one would then also expect that the contribution of three-body forces 35 to the binding energy per particle in finite nuclei continues to be slightly attractive when particle number is increased substantially beyond 10.36 This point and the previous discussion also suggest that there would be no further need for the ad-hoc repulsion added to three-body forces used to fit nuclear-matter saturation properties.37

\section{Conclusions}

One of the critical experimental ingredients in clarifying the nature of nuclear correlations has only become available over the last decade and a half. It is therefore not surprising that all schemes that have been developed to calculate nuclear-matter saturation properties are not based on the insights that these experiments provide. One of the aims of the present paper is to remedy this situation. To this end we have started with a brief reminder of experimental data obtained from the (e,e'p) reaction and corresponding theoretical results, that exhibit clear evidence that nucleons in nuclei exhibit strong correlation effects. Based on these considerations and the success of the theoretical calculations to account for the qualitative features of the sp strength distributions, it is suggested that the dressing of nucleons must be taken into account in calculations of the energy per particle. By identifying the dominant contribution of SRC to the empirical saturation density, it is argued that these correlations need to be emphasized in the study of nuclear matter. It is also argued that inclusion of long-range correlations, especially those involving pion propagation, leads to an unavoidable increase in the theoretical saturation density. Since this collectivity in the pion channel is not observed in nuclei, it is proposed that the corresponding correlations in nuclear matter are not relevant for the study of nuclear saturation and should therefore be excluded from consideration. A scheme which fulfills this requirement and includes the propagation of dressed particles, as required by experiment, is oytlined. Successful implementation of this scheme has recently been demonstrated.19.27 First results demonstrate that these new calculations lead to substantially lower saturation densities than have been obtained in the past. The introduction of a "nuclear-matter problem" which focuses solely on the contribution of SRC may therefore lead to new insight into the long-standing problem of nuclear saturation.

\section{Acknowledgments}

This work was supported by the U. S. National Science Foundation under Grant No. PHY-9900713.

manch1: submitted to World Scientific on October 24, 2018 


\section{References}

1. L. Lapikás, Nucl. Phys. A553, , (2)97c (1993).

2. V. R. Pandharipande, I. Sick, and P. K. A de Witt Huberts, Rev. Mod. Phys. 69, 981 (1997).

3. W. H. Dickhoff and H. Müther, Rep. Prog. Phys. 55, 1947 (1992).

4. H. Müther and W. H. Dickhoff, Phys. Rev. C 49, R17 (1994).

5. H. Müther, A. Polls, and W. H. Dickhoff, Phys. Rev. C 51, 3040 (1995).

6. V.M. Galitski and A.B. Migdal, Sov. Phys. JETP 34, 96 (1958).

7. D. S. Koltun, Phys. Rev. C9, 484 (1974).

8. I. Sick and P. K. A. de Witt Huberts, Comm. Nucl. Part. Phys. 20, 177 (1991).

9. P. Grabmayr, Prog. Part. Nucl. Phys. 29, 251 (1992).

10. L. Lapikás, private communications (2000 and 2001).

11. M. F. van Batenburg, Ph. D. Thesis, University of Utrecht, 2001.

12. G. A. Rijsdijk, K. Allaart, and W. H. Dickhoff, Nucl. Phys. A550, 159 (1992).

13. S. Fantoni and V. R. Pandharipande, Nucl. Phys. A427, 473 (1984).

14. B. E. Vonderfecht et al., Phys. Rev. C 44, R1265 (1991).

15. B. Frois et al., Phys. Rev. Lett. 38, 152 (1977).

16. H. Q. Song et al., Phys. Rev. Lett. 81, 1584 (1998).

17. B. E. Vonderfecht et al., Nucl. Phys. A555, 1 (1993).

18. D. Rohe, in Proc. Fifth Workshop Electromagnetically induced two-nucleon emission, eds. P. Grabmayr et al. (University of Lund, published on CD) in press.

19. E. P. Roth, Ph. D. Thesis, Washington University, St. Louis, 2000.

20. A. Ramos, Ph.D. Thesis, University of Barcelona, 1988.

21. A. Ramos, A. Polls, and W. H. Dickhoff, Nucl. Phys. A503, 1 (1989).

22. R. V. Reid, Ann. of Phys. 50, 411 (1968).

23. B. E. Vonderfecht, Ph.D. Thesis, Washington University, St. Louis, 1991.

24. C. C. Gearhart, Ph.D. Thesis, Washington University, St. Louis, 1994.

25. W. H. Dickhoff et al., Phys. Rev. C 60, 064319 (1999).

26. Y. Dewulf, Ph.D. Thesis, University of Gent, 2000.

27. Y. Dewulf, D. Van Neck, and M. Waroquier, in preparation.

28. B. D. Day and R. B. Wiringa, Phys. Rev. C32, 1057 (1985).

29. A. D. Jackson, A. Landé, and R. A. Smith, Phys. Rep. 86, 55 (1982).

30. W. H. Dickhoff, A. Faessler, and H. Müther, Nucl. Phys. A389, 492 (1982).

31. B. D. Day, Phys. Rev. C 24, 1203 (1981).

32. W. H. Dickhoff, Prog. Part. Nucl. Phys. 12, 529 (1983).

33. P. Czerski, H. Müther, and W. H. Dickhoff, J. Phys. G: Nucl. Part. Phys. 20, 425 (1994).

34. P. Czerski, et al., Phys. Rev. C33, 1753 (1986).

35. S. C. Pieper et al., Phys. Rev. C 64, 014001 (2001).

36. R. B. Wiringa et al., Phys. Rev. C 62, 014001 (2000).

37. J. Carlson, V. R. Pandharipande, and R. B. Wiringa, Nucl. Phys. 401, 59 (1983).

manch1: submitted to World Scientific on October 24, 2018 